Relations industrielles

Industrial Relations

\title{
Le devoir de représentation et les associations de salariés depuis l'entrée en vigueur du projet de loi 45 .
}

\section{Louis Garant}

Volume 35, numéro 3, 1980

URI : https://id.erudit.org/iderudit/029098ar

DOI : https://doi.org/10.7202/029098ar

Aller au sommaire du numéro

Éditeur(s)

Département des relations industrielles de l'Université Laval

ISSN

0034-379X (imprimé)

1703-8138 (numérique)

Découvrir la revue

Citer cet article

Garant, L. (1980). Le devoir de représentation et les associations de salariés depuis l'entrée en vigueur du projet de loi 45. Relations industrielles / Industrial Relations, 35(3), 570-578. https://doi.org/10.7202/029098ar

Tous droits réservés (C Département des relations industrielles de l'Université Laval, 1980
Ce document est protégé par la loi sur le droit d'auteur. L'utilisation des services d'Érudit (y compris la reproduction) est assujettie à sa politique d'utilisation que vous pouvez consulter en ligne.

https://apropos.erudit.org/fr/usagers/politique-dutilisation/ 


\title{
Le devoir de représentation et les associations de salariés depuis l'entrée en vigueur du projet de loi 45
}

\author{
Louis Garant
}

Le 22 décembre 1977, l'Assemblée nationale du Québec adoptait le projet de loi 45 visant à modifier le Code du travail. Par cette loi qui est devenue le chapitre 41 des statuts de 1977, le législateur introduisait dans le Code du travail certains articles faisant état du devoir de représentation d'une association de salariés. C'est aux articles $38 \mathrm{~b}$ à $38 \mathrm{f}$ que l'on peut retrouver ces nouvelles dispositions qui se lisent comme suit:

38b. Une association accréditée ne doit pas agir de mauvaise foi ou de manière arbitraire ou discriminatoire, ni faire preuve de négligence grave à l'endroit des salariés compris dans une unité de négociation qu'elle représente, peu importe qu'ils soient ses membres ou non.

1977 , c. 41 , a. 28.

38c. Si un salarié qui a subi un renvoi ou une sanction disciplinaire croit que l'association accréditée viole à cette occasion l'article $38 \mathrm{~b}$, il doit, s'il veut se prévaloir de cet article, porter plainte par écrit au ministre dans les six mois. Le ministre nomme un enquêteur qui tente de régler la plainte à la satisfaction de l'intéressé et de l'association accréditée. 1977, c. 41, a. 28.

38d. Si aucun règlement n'intervient dans les quinze jours de la nomination de l'enquêteur ou si l'association ne donne pas suite à l'entente, le salarié doit, s'il veut se prévaloir de l'article 38b, faire une requête au tribunal dans les quinze jours suivants et demander à ce dernier d'ordonner que sa réclamation soit déférée à l'arbitrage.

1977 , c. 41, a. 28.

38e. Si le tribunal estime que l'association a violé l'article $38 \mathrm{~b}$, il peut autoriser le salarié à soumettre sa réclamation à un arbitre nommé par le ministre pour décision selon la convention collective comme s'il s'agissait d'un grief. Les articles 88 à $89 \mathrm{j}$ s'appliquent, mutatis mutandis. L'association paie les frais encourus par le salarié.

Le tribunal peut, en outre, rendre toute autre ordonnance qu'il juge nécessaire dans les circonstances.

1977, c. 41, a. 28.

- GARANT, Louis, avocat, Centre de recherche et de statistiques sur le marché du travail, ministère du Travail et de la Main-d'oeuvre, Québec. 
38f. Si une réclamation est déférée à un arbitre en vertu de l'article 38e, l'employeur ne peut opposer l'inobservation par l'association de la procédure et des délais prévus à la convention collective pour le règlement des griefs.

1977, c. 41, a. 28.

\section{LES ORIGINES DES ARTICLES 38b À 38f DU CODE DU TRAVAIL}

\section{Aux États-Unis}

Le devoir de représentation du syndicat est une doctrine qui a pris naissance aux Etats-Unis et la Cour suprême de ce pays en a clarifié et défini le contenu.

Un des premiers arrêts de jurisprudence faisant état de cette doctrine est: Steele v. Louisville and Nashville Railroad, 323 U.S. 192 (1944). Dans cette affaire, il s'agissait d'une question de discrimination raciale contre les noirs. En effet, une association de salariés voulait graduellement éliminer les travailleurs noirs du groupe de salariés représentés. Le tribunal saisi du litige a statué que le pouvoir exclusif de représentation du syndicat, conféré par le Railway Labor Act impliquait aussi le devoir de représenter tous les salariés inclus dans l'unité de négociation, qu'ils soient membres ou non de l'association de salariés. De plus, ce devoir doit se faire:

fairly, impartially and in good faith... The language of the act... expresses the aim of Congress to impose on the bargaining representative of a craft or class of employees the duty to exercise fairly the power conferred upon it in behalf of all those for whom it acts, without hostile discrimination against them. ${ }^{1}$

Cette obligation statutaire de représenter tous les salariés a été étendue dans l'arrêt Conley v. Gibson'. La Cour suprême des États-Unis déclarait alors que le devoir du syndicat s'étendait à l'administration et l'application de la convention collective.

The bargaining representative's duty not to draw irrelevant and invidious distinctions among those it represents does not come to an abrupt end... with the making of an agreement between union and employer. Collective bargaining is a continuing process. Among other things, it involves... the protection of employee rights already secured by contract. ${ }^{3}$

L'étape la plus significative quant à l'évolution de cette théorie est survenue avec l'arrêt $\operatorname{Vaca}$ v. Sipes ${ }^{4}$.

1 Steele v. Louisville and Nashville Railroad, 323 U.S. 192 (1944), pp. 202-204.

355 U.S. 41 (1957).

3 Ibid., p. 46.

4386 U.S. 171 (1967). 
Dans cette affaire, un salarié poursuivait son association parce que cette dernière n'avait pas poussé sa plainte à l'arbitrage. La prétention du salarié était qu'il avait été injustement congédié.

La Cour suprême des États-Unis a alors déclaré, dans son jugement, qu'une association de salariés ne pouvait agir arbitrairement et ignorer le grief d'un salarié, ni agir uniquement pour la forme (merely perfunctorily). Toutefois un salarié n'a pas un droit automatique de voir son grief déféré à l'arbitrage, ce droit est subordonné aux dispositions de la convention collective. De plus, l'association de salariés peut «régler» un grief avant arbitrage ou refuser de l'y amener (settle or dispose). Dans ces cas, l'association doit agir sans commettre de gestes arbitraires, discriminatoires ou de mauvaise foi.

Au Canada

Les lois canadiennes du travail introduisirent beaucoup plus tard cette notion importée de la loi américaine. Cependant la jurisprudence des tribunaux supérieurs du pays introduisit certaines notions dès le milieu des années soixante. En effet dans l'arrêt Dirk Hoogendoorn v. Greening Metal Products and Screening Equipment Company and the United Steelworkers of America, local 6266, 5 la Cour suprême du Canada déclarait que la justice naturelle exigeait que le plaignant soit représenté et entendu. La Cour citait à ce titre certaines décisions du Conseil privé de Londres établissant les règles relatives au droit d'être entendu (audi alteram partem).

Les tribunaux canadiens ont eu aussi l'occasion de se prononcer lors de décisions impliquant des dommages-intérêts. Ainsi dans l'affaire Fisher v. Pemberton ${ }^{6}$ la Cour suprême de la Colombie-Britannique statuait que:

"Certainly in this case the local union did not make in a non-arbitrary manner a decision as to the merits of Fisher's grievance. The whole matter was handled in a perfunctory way. I conclude therefore that the plaintiff has proved against local 592 the breach of duty charged."

C'est donc ainsi qu'en février 1971 les lois ontariennes furent amendées pour incorporer une disposition législative spécifique qui cristallisa la jurisprudence américaine et canadienne sur le sujet.

La législature québécoise en fit tout autant lors de l'adoption du projet de loi 45 modifiant le Code du travail du Québec ${ }^{7}$ le 22 décembre 1977.

5 (1968) R.C.S., p. 30.

6 (1970) 8 D.L.R., p. 521.

7 L.Q. 1977 , c. 41 , a. 28. 


\section{QUELQUES STATISTIQUES CONCERNANT L'APPLICATION} DES ARTICLES 38b à $38 \mathrm{f}$

Concernant le nombre de plaintes faites au ministre, nous constatons qu'aucune n'a été faite avant le mois de septembre 1978, malgré que les articles $38 \mathrm{~b}$ et suivants aient été en vigueur depuis février 1978. Ce fait est sans doute dû au délai nécessaire pour que le public prenne connaissance de la loi.

Lorsque nous comparons le nombre de plaintes déférées à un enquêteur (294) et celles adressées au Tribunal du travail (118), nous constatons que seulement $40 \%$ des premières arrivent à la seconde étape. De plus, seulement 11 jugements ont autorisé que la plainte du salarié soit déférée à l'arbitrage. Il nous est cependant impossible d'évaluer le nombre de cas qui se sont réglés grâce à l'intervention de l'enquêteur ou qui ont fait l'objet d'un règlement à l'amiable après que la plainte eut été faite au ministre. Nous tenons aussi à signaler le nombre élevé de désistements, un règlement ayant pu intervenir dans certains de ces cas.

\section{Tableau 1}

Plaintes déférées à un enquêteur par le ministre du Travail, selon l'article 38c

$\begin{array}{lrrrl} & 1978 & 1979 & 1980 & \\ \text { Janvier } & & 6 & 42 & \\ \text { Février } & & 22 & 14 & \\ \text { Mars } & & 12 & 20 & \\ \text { Avril } & & 17 & & \\ \text { Mai } & & 19 & & \\ \text { Juin } & & 15 & & \\ \text { Juillet } & & 18 & & \\ \text { Aoat } & 8 & 6 & & \text { Grand total 294 } \\ \text { Septembre } & 11 & 10 & & \\ \text { Octobre } & 16 & 12 & & \\ \text { Novembre } & 11 & 19 & & \\ \text { Décembre } & 46 & 172 & 76 & \\ \text { TOTAL } & & & & \end{array}$

Tableau 2

Requêtes adressées au Tribunal du travail selon l'article 38d (selon les statistiques fournies par le greffe du tribunal)

Jusqu'en date du mois de mai 1980

$\begin{array}{lr}\text { rejetées } & 22 \\ \text { accueillies } & 11 \\ \text { désistements } & 19 \\ \text { pendantes } & 66 \\ \text { total } & 118\end{array}$




\section{ETUDE DE LA JURISPRUDENCE QUÉBECCISE}

Renvoi ou sanction disciplinaire

Une plainte ne peut être adressée au Tribunal du travail que dans les cas de renvoi ou de sanction disciplinaire. La compétence du tribunal, en relation avec l'application de l'article $38 \mathrm{~b}$, ne peut s'étendre à tout geste arbitraire ou discriminatoire posé par l'association de salariés.

«L'article $38 \mathrm{~b}$ édicte une prohibition de nature générale; il impose à une association accréditée l'obligation de ne pas agir de mauvaise foi, de manière arbitraire ou discriminatoire, etc... à l'endroit des salariés compris dans une unité de négociation qu'elle représente. Cette prohibition permettrait peut-être les recours prévus à l'article 126 C.t., mais lorsqu'il s'agit de rechercher un remède approprié en faveur d'un salarié victime du refus de l'association de se conformer à ses obligations, l'éventail des remèdes est limité aux cas de renvoi ou de sanction disciplinaire. ${ }^{8}$

Toutefois, le tribunal a considéré que le non-renouvellement d'un contrat d'enseignant était assimilable à un renvoi. ${ }^{9}$

Mauvaise foi, manière arbitraire ou discriminatoire, négligence grave

FARDEAU ET DEGRE DE PREUVE

Il importe au salarié de prouver que l'association a agi à l'encontre de ses obligations et le tribunal doit constater prima facie que les motifs allégués pour le congédiement paraissent fondés. Il ne s'agit pas de remplacer l'arbitre et de porter un jugement sur le grief. ${ }^{10}$

Le Tribunal du travail se prononçait ainsi sur ce sujet:

«...le fardeau du requérant ne vas pas jusqu'à exiger qu'il fasse la preuve que son grief est bien fondé ou aurait quelque chance de succès s'il était mené à l'arbitrage... c'est avant tout à l'examen du processus de décision suivi par l'association de salariés que s'attachera le tribunal.."11

Par la suite le tribunal ajoutera qu'il suffit de constater que prima facie, la prétention du requérant à l'égard de sa plainte est sérieuse, non futile et plausible pour accorder la requête ${ }^{12}$.

8 Réjeanne Baker c. Syndicat Canadien de la fonction publique et Ville de Varennes (1978) T.T., p. 445.

9 Bordeleau c. Syndicat des professeurs du Collège du Nord-ouest et Collège du Nordouest (1979) T.T., p. 133.

10 Voir dans ce sens: Yvon Bibeau c. Fraternité unie des charpentiers et menuisiers d'Amérique et ATCO Métal Ltd. (1978) T.T., p. 131.

11 Adrien Boulay c. Fraternité des policiers de la C.U.M. et le conseil de sécurité de la C.U.M. (1978) T.T., p. 319.

12 Claude Maurice c. Union Canadienne des travailleurs unis de brasseries et O'Keefe Ltée (1979) T.T., p. 82. 
Nous avons vu que le tribunal considère que le fardeau de la preuve repose sur le salarié, nous allons maintenant aborder la question de la nature de l'infraction ou en d'autres termes, ce qui constitue une infraction aux articles $38 \mathrm{~b}$ et suivants.

La notion civiliste du «bon père de famille» a été appliquée par le Tribunal du travail pour qualifier la conduite d'une association de salariés face à son membre.

«...le président, tant dans les efforts qu'il apporta pour faire comprendre à monsieur Bibeau l'importance de la clause d'ancienneté pour tous les salariés que dans ses démarches pour convaincre monsieur Bibeau de représenter au comité son point de vue, a démontré qu'il se comportait comme un bon père de famille, expliquant à un membre de l'association ses droits et l'invitant à les faire valoir selon les procédures établies. $)^{13}$

Nous pouvons donc référer à cette notion pour dire que l'association n'a pas contrevenu à son obligation de représentation si elle s'est comportée en bon père de famille dans ses agissements face aux revendications du salarié.

Par ailleurs le tribunal a déjà considéré que le simple refus de procéder à grief et arbitrage sans raison valable est arbitraire et qu'en matière de congédiement, le fait de ne pas apporter un soin raisonnable à la protection de l'emploi d'un salarié pouvait être une négligence grave ${ }^{14}$. Le juge Brière mentionnait à ce titre:

«C'est dans l'ensemble du fait et de ses effets, de la faute et de ses conséquences, de la cause et du préjudice en résultant, qu'il y a lieu d'apprécier le droit. C'est pourquoi je n'hésite pas à dire qu'une faute légère (par exemple, un simple oubli) qui entraîne la perte d'un recours à l'arbitrage susceptible de conserver à un salarié son emploi constitue véritablement une négligence grave. $)^{15}$

La jurisprudence a aussi considéré que l'association de salariés, comme corollaire à son monopole de représentation, doit exercer ses responsabilités...

«dont elle avait voulu, à l'avantage des salariés, se charger aux termes de la convention collective. Son défaut de se donner des représentants pour fin de la rencontre "obligatoire» avec le mis en cause (l'employeur) avant que ce dernier ne prenne une décision relative au requérant constituait une négligence des plus graves.»16

13 Yvon Bibeau c. Fraternité unie des charpentiers et menuisiers d'Amérique et ATCO Métal Ltée (1978) T.T., p. 131.

14 Daniel Courchesne c. le Syndicat des travailleurs de la Corporation de Batteries CEGELEC et la Corporation de Batteries CEGELEC (1978) T.T., p. 328. Voir aussi: Guenette c. l'Union des chauffeurs de camions, local 106 et $H$. Smith Transport Ltée (1979) T.T., p. 409.

15 Décision Courchesne (1978) T.T., pp. 328-335.

16 Bordeleau c. Syndicat des professeurs du Collège du Nord-ouest et Collège du Nord- 
La bonne foi est un élément important dans l'attitude de l'association de salariés, mais toutefois elle ne saurait être le motif principal puisque le Tribunal du travail a déjà déclaré que l'apparente bonne foi des dirigeants syndicaux ne pouvait bonifier l'attitude négligente avec laquelle ils ont traité le grief d'un salarié ${ }^{17}$. En effet dans ce cas, l'association n'avait pas fait une enquête sérieuse pour se convaincre des prétentions du requérant.

Ce devoir de représentation attribué au syndicat ne doit pas être limité dans le temps:

"Cette obligation doit être respectée dans tous les actes, subséquents à la mesure disciplinaire, que le syndicat est appelé à poser pour le salarié y compris lors du déroulement de l'arbitrage. ${ }^{18}$

Il peut arriver que le syndicat pose des gestes qui seront néfastes au salarié en cause sans toutefois commettre ces gestes de mauvaise foi ou avec discrimination. Les critères retenus par la jurisprudence pour évaluer cette situation sont que l'obligation de juste représentation sera considérée respectée en l'absence de preuve d'intention frauduleuse et malveillante. S'il est démontré que les démarches du syndicat se sont déroulées sous le signe de la bonne foi et furent dénuées d'attitudes arbitraires, le salarié n'aura pas de recours contre son association ${ }^{19}$. Cependant, le syndicat ne doit pas faire preuve de négligence grave auquel cas il ne pourra échapper à un jugement condamnant son attitude, même s'il se replie sur sa bonne foi et son absence d'intention frauduleuse ${ }^{20}$.

Ainsi il fut décidé qu'une association n'avait pas manqué à son devoir de représentation en interprétant mal la convention collective au chapitre des délais pour soumettre un grief. Il s'agissait d'une interprétation tout à fait raisonnable que l'arbitre de grief avait longuement analysée avant de la rejeter ${ }^{21}$. Il s'agissait donc d'une erreur due au manque d'expérience des représentants syndicaux, erreur n'équivalant pas à négligence grave. Dans ce même jugement, le juge Saint-Arnaud déclarait que la négligence grave doit s'apparenter...

«à une attitude marquée soit par l'erreur grossière, par la faute lourde commise par ses représentants, par une omission impardonnable des précautions requises, par un manque d'habilité notoire et évident par une insouciance manifeste dénotant l'incapacité de cette association de prendre sérieusement et efficacement en mains les intérêts des salariés compris dans son unité de négociation. ${ }^{22}$

ouest (1979) T.T., pp. 133-139.

17 Johanne Boutin c. Syndicat International des travailleurs en électricité, de radio et de machinerie et CAE Electronics Ltée (1979) T.T., p. 91.

18 Jacques Bécotte c. Syndicat Canadien de la fonction publique et Association récréative et culturelle (1979) T.T., p. 231.

19 Ibid., p. 235 où l'on cite la jurisprudence américaine sur le sujet.

20 Idem.

21 Ibid., p. 237.

22 Ibid., p. 236. 
Il est toutefois difficile de bien identifier les motifs à alléguer dans le cas de violation de l'article $38 \mathrm{~b}$.

«le requérant devra établir... la mauvaise foi, l'arbitraire, la discrimination ou encore la négligence grave... La distinction à faire entre ces trois expressions m'apparaît assez difficile. Cependant on peut y reconnaître certaines modalités ou différences selon les faits prouvés.» ${ }^{23}$

\section{L'ATTITUDE ET LE COMPORTEMENT DU PLAIGNANT}

L'association de salariés, dans l'exercice de son devoir de représentation peut faire face à diverses difficultés résultant des agissements mêmes des salariés. L'attitude du plaignant est donc d'une grande importance car elle influence le déroulement de ses revendications. Il doit afficher une attitude de collaboration et ne pas oublier que l'association a discrétion dans l'acheminement de son grief.

«le salarié n'a pas un droit absolu à l'arbitrage, ni un droit absolu à ce que son association agisse selon ses instructions; il a cependant un droit clairement établi - et c'est là, la protection que lui accorde la loi - à ce que son association n'abuse pas de sa discrétion en agissant de mauvaise foi, discriminatoirement, arbitrairement ou en étant gravement négligente, lorsqu'il lui demande de déposer un grief en son nom. ${ }^{24}$

Le salarié qui refuserait un règlement raisonnable de son grief et qui afficherait une attitude intransigeante à l'égard des représentants syndicaux tout en refusant de répondre à leurs questions verrait sa plainte rejetée par le tribunal sans hésitation ${ }^{25}$. En effet, pour que sa plainte soit accueillie, le salarié doit, en plus de prouver l'attitude fautive de son association, démontrer avoir affiché un comportement de collaboration. C'est le cas du salarié qui se désintéresse de son grief, agissant avec négligence, en ne rencontrant pas les officiers syndicaux lorsque ceux-ci sont disponibles ${ }^{26}$.

Sur ce sujet le tribunal déclarait:

«La collaboration dont il s'agit est celle que doit apporter à son syndicat le salarié qui fait un grief. Ce dernier doit alors être disponible pour rencontrer les dirigeants syndicaux et fournir avec diligence à son syndicat tous les renseignements concernant son grief ... l'expression «comportement de collaboration" ne peut nullement signifier qu'un salarié doit

23 Guenette c. l'Union des chauffeurs de camions, local 106 et $\boldsymbol{H}$. Smith Transport Ltée (1979) T.T., p. 409.

24 Boulay c. Fraternité des policiers de la C.U.M. et le Conseil de sécurité de la C.U.M. (1978) T.T., p. 319 et pp. 326-327.

25 À ce titre voir Godin c. Fraternité Unie des charpentiers local 2533 et Ideal Builders Hardware (1979) T.T., p. 157.

26 Drolet c. Syndicat des employés du supermarché Roy Inc. et Supermarché Roy Inc. (1979) T.T., p. 221. 
avoir des opinions ou des attitudes concordantes avec celles du syndicat et qu'il ne doit pas trop critiquer ce dernier.» ${ }^{27}$

L'association de salariés, après que l'individu eut apporté sa collaboration, ne peut refuser de le défendre si son grief est fondé,

«que ce dernier soit un contestataire, un activiste ou même un révolutionnaire, ou encore le partisan fanatique d'un syndicat rival... ${ }^{28}$

et le tribunal ajoute que l'association ne peut manquer à son devoir de représentation sous prétexte que le salarié aurait quelquefois violé les statuts du syndicat.

\title{
CONCLUSION
}

L'introduction des articles $38 \mathrm{~b}$ à $38 \mathrm{f}$ dans le Code du travail du Québec s'inscrit, selon les débats de la commission permanente du travail, de la main-d'oeuvre et de l'immigration du 17 décembre 1977, dans une attitude de confiance à l'égard des associations accréditées. Selon les paroles du ministre Johnson, la démocratie syndicale est la règle générale au Québec. Cependant, il faut que la loi impose des balises pour que chaque association ait les mêmes responsabilités, tout en conservant la façon la plus adéquate de les appliquer pour le bénéfice des salariés.

L'étude de ces jugements du Tribunal du travail nous a démontré que ce ne sont pas les notions fixes et immuables qu'il faut respecter mais bien une philosophie, une manière d'agir et des règles de conduite. C'est à chaque fois un cas d'espèce qui survient, cas que nous devons adapter à un autre avec beaucoup de circonspection. Ce ne sont pas des faits pris isolément, hors-contexte qu'il faut retenir mais bien l'idée qui se dégage de chaque intervention du tribunal. Le devoir de représentation est un principe au même titre que la responsabilité civile. Seule la jurisprudence peut en délimiter la portée dans le temps.

\section{L'octroi de dommages-intérêts par un tribunal d'arbitrage dans le cas d'une grève contraire à la convention collective}

\author{
Pierre Verge
}

\section{Par un arrêt unanime, la Cour Suprême du Canada renverse un courant jurisprudentiel bien établi de la Cour d'appel du}

* VERGE, Pierre, professeur, faculté de Droit, Université Laval.

27 Legault c. Syndicat des travailleurs amalgamés du vêtement et du textile et Morwill Clothing et Stylemart Clothing (1979) T.T., p. 375 et p. 385.

28 Ibid., p. 384. 